\title{
Effect of cycloheximide on nuclear maturation of horse oocytes and its relation to initial cumulus morphology
}

\author{
H. Alm ${ }^{1}$ and K. Hinrichs ${ }^{2}$ \\ ${ }^{1}$ Department of Reproductive Biology, Research Institute for the Biology of Farm Animals, 18196 \\ Dummerstorf, Germany; and ${ }^{2}$ Tufts University School of Veterinary Medicine, 200 Westboro Road, \\ North Grafton, MA 01536, USA
}

\begin{abstract}
The period of protein synthesis necessary for meiotic maturation in horse oocytes initially having compact or expanded cumulus cells was studied. Oocytes incubated in the presence of cycloheximide after $0,4,8,12$ or $16 \mathrm{~h}$ maturation in vitro (total incubation time $24 \mathrm{~h}$ ) were matured for $24 \mathrm{~h}$, or were incubated with cycloheximide for $24 \mathrm{~h}$ and then matured for $24 \mathrm{~h}$. Incubation with cycloheximide from $\mathrm{O} h$ was effective in suppressing maturation (no significant increase in maturing oocytes compared with controls fixed directly after removal from the follicle) in both expanded and compact groups and was completely reversible, as there was no difference in the proportion of oocytes reaching metaphase II between controls and treatment groups of either cumulus type. Addition of cycloheximide after $4 \mathrm{~h}$ maturation resulted in no significant difference in distribution of oocytes compared with addition at $\mathrm{O} h$ in either cumulus group. A significant decrease in the proportion of germinal vesicle stage oocytes, and an increase in oocytes in metaphase I occurred in oocytes with expanded cumulus cells in the $8 \mathrm{~h}$ treatment and in oocytes with compact cumulus cells in the $12 \mathrm{~h}$ treatment, compared with oocytes treated after $\mathrm{O}$ h. A significant increase in the proportion of oocytes at metaphase II occurred in the $12 \mathrm{~h}$ treatment for expanded cumulus-oocyte complexes and in the $16 \mathrm{~h}$ treatment for compact cumulus-oocyte complexes. These data show that nuclear maturation of horse oocytes can be reversibly suppressed by incubation with cycloheximide from the onset of culture. Oocytes with different initial cumulus type differed in the time required for protein synthesis essential for maturation: expanded cumulus-oocyte complexes required less time to prepare for germinal vesicle breakdown, maturation to metaphase I, and maturation to metaphase II, than did compact cumulus-oocyte complexes.
\end{abstract}

\section{Introduction}

Fully grown oocytes of mammalian species are arrested in prophase I of meiosis until the preovulatory surge of $\mathrm{LH}$, or release from the follicle in vitro (Pincus and Enzmann, 1935). Resumption of oocyte maturation involves both synthesis and phosphorylation of proteins. Cycloheximide, which inhibits peptidyl transferase, suppresses oocyte maturation by blocking synthesis of stage-specific proteins. In pigs, the pattern of proteins synthesized, as visualized by gel electrophoresis, changes significantly before germinal vesicle breakdown (GVBD; McGaughey and Van Blerkom, 1977). In cows, although no protein changes are seen in the protein synthesis pattern of oocytes before GVBD (Kastrop et al., 1990), culture of oocytes in the presence of cycloheximide inhibits protein phosphorylation (Kastrop et al., 1991). This led these authors to hypothesize that small amounts of enzymes involved in phosphorylation of existing proteins must be synthesized for GVBD to occur.

Received 5 January 1996.
The period of protein synthesis essential for the resumption of the first meiotic division appears to be related to the period needed for GVBD and maturation. Mouse oocytes take 12-14 $\mathrm{h}$ to mature and GVBD occurs within $2 \mathrm{~h}$ (Donahue, 1968); treatment with cycloheximide from the onset of culture does not prevent GVBD. Bovine oocytes take $24 \mathrm{~h}$ to mature and GVBD occurs within 6-9 h (Sirard and First, 1988; Sirard et al., 1989); treatment with cycloheximide up to $6 \mathrm{~h}$ after the onset of maturation prevents GVBD (Sirard, 1990; Kastrop et al. 1991; Tatemoto and Terada, 1995). Pig oocytes take $44 \mathrm{~h}$ to mature and GVBD occurs after approximately $16-20 \mathrm{~h}$ (Motlik and Fulka, 1976); GVBD occurred in 15, 46 and $75 \%$ of oocytes when cycloheximide was added after 6,12 and $16 \mathrm{~h}$ of culture, respectively (Fulka et al., 1986). Maturation to metaphase II requires synthesis of additional new proteins. In mice, while oocytes cultured in the presence of protein synthesis inhibitors undergo GVBD, they do not proceed beyond metaphase I (Stern et al., 1972; Wassarman et al., 1976; Schultz and Wassarman, 1977). In bovine oocytes, when cycloheximide is added after $9 \mathrm{~h}$ of maturation, essentially all oocytes undergo GVBD (Sirard et al., 1989; Kastrop et al., 
1991). However, maturation to metaphase II occurs only if cycloheximide is added after $12 \mathrm{~h}$ of culture. Little work has been done on meiotic arrest of horse oocytes. Hinrichs et al. (1995a) reported that follicular fluid alone did not suppress maturation, but follicle wall tissue (sheets of granulosa, follicle wall sections or intact follicle) did maintain oocytes in the germinal vesicle stage. Differences in viability (maturation to metaphase II) after suppression were found between oocytes originally having compact (CP) or expanded (EX) cumulus cells, with EX oocytes maturing in significantly higher proportions.

Previous studies have shown that although EX oocytes are recovered mainly from atretic follicles (Hinrichs, 1991), there is no difference in the proportion originally at the germinal vesicle stage when compared with CP oocytes (Hinrichs et al. 1993a), and there is no difference in the ability of EX and CP oocytes to mature to metaphase II (Zhang et al., 1989; Hinrichs et al., 1993a). However, the time sequence of maturation is different for EX and CP oocytes. Significantly more EX than CP oocytes had undergone GVBD at $8 \mathrm{~h}$ and at $16 \mathrm{~h}$ of culture, and maximum maturation occurred at $24 \mathrm{~h}$ for EX oocytes but at $32 \mathrm{~h}$ for CP oocytes (Hinrichs et al., 1993a).

This study was designed to investigate whether maturation of horse oocytes is suppressed by culture with cycloheximide, and the degree to which such suppression is reversible. In addition, we wished to define the period of protein synthesis necessary for meiotic maturation in horse oocytes, and to determine whether the timing of this critical period varies with different initial cumulus morphology.

\section{Materials and Methods}

\section{Experimental design}

Oocytes were assigned to one of eight groups: direct control (Dir), fixed directly upon removal from the follicle; maturation control (Mat), culture in maturation medium for $24 \mathrm{~h}$; suppression groups, matured in vitro for $0,4,8,12$ or $16 \mathrm{~h}$, and then transferred to cycloheximide-containing medium - total incubation time $24 \mathrm{~h}$; or suppression followed by maturation (Cyc-Mat), culture in the presence of cycloheximide for $24 \mathrm{~h}$ and then transfer to maturation medium for a further $24 \mathrm{~h}$. Each treatment was carried out for each cumulus type (CP and EX). Oocytes (12 of each cumulus type per replicate) were assigned to the eight treatments in randomized order. Each replicate of eight groups was randomized separately. If at the end of a collection day fewer than 12 oocytes were available for one treatment, the replicate was filled on the next collection day. The experiment was continued until there was a minimum of 30 analysable oocytes and three replicates in each treatment.

\section{Methods}

Horse ovaries were obtained from a local abattoir, placed in saline containing $0.1 \mathrm{mg}$ ticarcillin $\mathrm{ml}^{-1}$ (Smith Kline Beecham Pharmaceuticals, Philadelphia, PA), and transported ( $1 \mathrm{~h}$ ) to the laboratory at room temperature. Oocytes were collected from all follicles of under $30 \mathrm{~mm}$ diameter. Oocytes were recovered by opening the follicles visible on the surface with a scalpel blade and scraping the interior of follicles with a bone curette (Hinrichs et al., 1993a). After all visible follicles were opened, the ovaries were cut into $5 \mathrm{~mm}$ sections to locate any follicles within the ovarian stroma. The scraped granulosa cells thus collected were washed into individual Petri dishes using modified M199 (M199 with Hank's salts and 25 mmol Hepes buffer $!^{-1}$ (Gibco Life Technologies, Inc., Grand Island, NY) and $0.1 \mathrm{mg}$ ticarcillin $\mathrm{ml}^{-1}$ ).

Cumulus-oocyte complexes were located using a dissecting microscope at a magnification of $\times 10$, and were classified as having compact (CP) or expanded (EX) cumulus cells (Fig. 1). The cumulus-oocyte complexes were dissected from attached mural granulosa and held at room temperature in modified M199 until incubation (maximum $2.5 \mathrm{~h}$ ). Oocytes without cumulus or with only corona radiata were not used in the experiments.

For chromatin evaluation, cumulus cells were removed by pipetting in a $0.25 \%(\mathrm{v} / \mathrm{v})$ solution of trypsin and $1 \mathrm{mmol} \mathrm{l}^{-1}$ EDTA in Hank's salt solution without $\mathrm{CaCl}_{2}, \mathrm{MgCl}_{2}$ or $\mathrm{MgSO}_{4}$ (Gibco Life Technologies, Inc.). The denuded oocytes were fixed for at least $24 \mathrm{~h}$ in buffered formol saline, and then placed on a slide and covered with $6 \mu \mathrm{l}$ mounting medium (3:1 glycerol:PBS) containing $2.5 \mu \mathrm{g}$ Hoechst $33258 \mathrm{ml}^{-1}$ (Hinrichs et al., 1993b). The oocytes were examined using a fluorescence microscope with a $G 365 \mathrm{~nm}$ exciter filter. The chromatin was classified as fluorescent nucleus (FN), diffuse fluorescence throughout the germinal vesicle; condensed chromatin (CC), the chromatin condensed in one mass (within the germinal vesicle, as shown by Hinrichs et al. (1993a)); crinkled fluorescent nucleus, the germinal vesicle was fluorescent but was misshapen, with a granular or crosshatched appearance; diakinesis (D), metaphase I, metaphase II or degenerating (chromatin spread throughout oocyte, abnormal chromatin or no chromatin visible) as described by Hinrichs et al. (1993b). Oocytes classified as $\mathrm{FN}$ or $\mathrm{CC}$ were considered to represent germinal vesicle stage oocytes (Hinrichs et al., 1993a).

For culture, cumulus-oocyte complexes were incubated in $200 \mu \mathrm{l}$ drops of maturation medium (M199 with Earle's salts supplemented with $10 \%$ neonatal calf serum, $25 \mu \mathrm{g}$ gentamycin $\mathrm{ml}^{-1}, 1.25 \mu \mathrm{g}$ Fungizone $\mathrm{ml}^{-1}$ (Gibco Life Technologies, Inc.) and $5 \mu \mathrm{g} \mathrm{FSH} \mathrm{ml}{ }^{-1}$ (Schering Corp. USA, Kenilworth, NI) under paraffin oil. The culture was carried out at $38.2^{\circ} \mathrm{C}$ in a humidified atmosphere of $5 \% \mathrm{CO}_{2}$. Inhibition treatments consisted of incubation in $200 \mu \mathrm{l}$ drops of the maturation medium containing $10 \mu \mathrm{g}$ cycloheximide $\mathrm{ml}^{-1}$ (Sigma Chemical Co., St Louis, MO).

\section{Statistical analyses}

Data were analysed using the chi-squared test with $P<0.05$ considered statistically significant. Fisher's exact test was used in comparisons in which fewer than five values were expected in any cell.

\section{Results}

From the 140 ovaries that were processed, 651 oocytes were recovered from 915 follicles, a $71.1 \%$ oocyte recovery rate. Of the collected oocytes, $330(50.7 \%)$ had a compact (CP) 

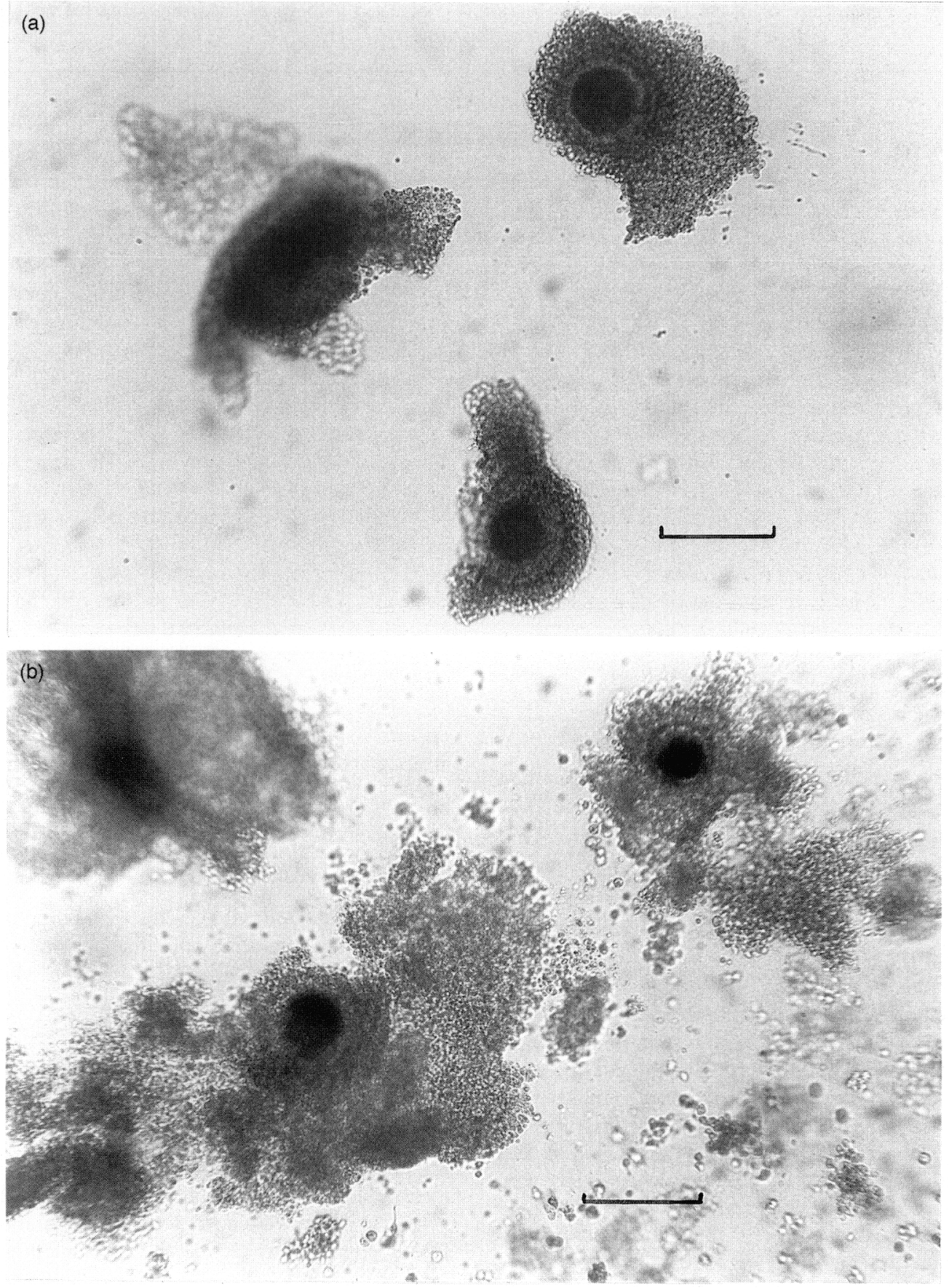

Fig. 1. Horse oocytes at the time of recovery. (a) Compact cumulus; (b) expanded cumulus. Scale bar represents $200 \mu \mathrm{m}$.

cumulus, $307(47.2 \%)$ had an expanded (EX) cumulus, and 14 $(2.2 \%)$ had no cumulus, corona radiata only, or had a visibly degenerated or damaged ooplasm. Only CP and EX oocytes ( $n=288$ for each cumulus type) were used in the study.

On evaluation of oocyte chromatin, we changed the classification system slightly from that used by Hinrichs et al. (1993a, b). Because the proportion of germinal vesicle stage oocytes that had the crinkled fluorescent nucleus configuration increased markedly over incubation, from $3 / 50$ for (combined EX and (P) directly fixed oocytes to $4 / 5$ for matured oocytes and $4 / 4$ for oocytes matured after suppression, we postulated that crinkled fluorescent nuclei are associated with abnormal or nonviable oocytes and placed this chromatin configuration in the degenerating category rather than with oocytes with fluorescent nuclei (germinal vesicle stage).

The distribution of oocytes among chromatin classifications in the different treatment groups is presented (Table 1 ). In direct controls, the proportion of oocytes in the germinal vesicle stage was not significantly different between $\mathrm{EX}$ and $\mathrm{CP}$ oocytes $(66 \%$ versus $67 \%$; $P>0.1)$. The proportion of germinal 
Table 1. Percentage of horse oocytes in the different chromatin categories according to treatment and cumulus type

\begin{tabular}{|c|c|c|c|c|c|c|c|c|}
\hline Treatment & $n$ & $\begin{array}{c}\text { Fluorescent } \\
\text { nucleus }\end{array}$ & $\begin{array}{l}\text { Condensed } \\
\text { chromatin }\end{array}$ & Diakinesis & Metaphase I & Metaphase II & $\begin{array}{l}\text { Crinkled } \\
\text { fluorescent } \\
\text { nucleus }\end{array}$ & Degenerating \\
\hline CP Direct & 36 & 25.0 & 41.7 & 2.8 & 0 & 0 & 2.8 & 27.7 \\
\hline EX Direct & 35 & 5.7 & 60.0 & 2.8 & 2.8 & 0 & 5.7 & 22.9 \\
\hline CPOh & 36 & 0 & 33.3 & 5.5 & 0 & 0 & 8.3 & 52.8 \\
\hline EXoh & 34 & 0 & 58.8 & 2.9 & 11.8 & 2.9 & 2.9 & 20.6 \\
\hline $\mathrm{CP} 4 \mathrm{~h}$ & 36 & 0 & 36.1 & 5.6 & 5.5 & 0 & 0 & 52.8 \\
\hline EX $4 \mathrm{~h}$ & 36 & 0 & 41.7 & 2.8 & 25.0 & 0 & 8.3 & 22.2 \\
\hline $\mathrm{CP} 8 \mathrm{~h}$ & 36 & 0 & 16.7 & 2.8 & 11.1 & 0 & 5.5 & 63.9 \\
\hline EX $8 \mathrm{~h}$ & 35 & 0 & 17.1 & 5.7 & 65.7 & 5.7 & 2.9 & 2.9 \\
\hline $\mathrm{CP} 12 \mathrm{~h}$ & 35 & 0 & 2.9 & 2.9 & 17.1 & 5.7 & 2.9 & 68.5 \\
\hline EX $12 \mathrm{~h}$ & 32 & 0 & 12.5 & 3.1 & 21.9 & 25.0 & 12.5 & 25.0 \\
\hline CP $16 \mathrm{~h}$ & 34 & 0 & 0 & 5.9 & 2.9 & 17.6 & 5.9 & 67.6 \\
\hline EX $16 \mathrm{~h}$ & 33 & 0 & 6.1 & 0 & 12.1 & 51.5 & 9.1 & 21.2 \\
\hline CP Mat & 34 & 0 & 2.9 & 0 & 5.9 & 20.6 & 2.9 & 64.7 \\
\hline EX Mat & 32 & 0 & 0 & 3.1 & 15.6 & 62.5 & 6.3 & 12.5 \\
\hline CP Cyc-Mat & 35 & 0 & 0 & 0 & 0 & 31.4 & 8.6 & 60.0 \\
\hline EX Cyc-Mat & 35 & 0 & 0 & 0 & 14.3 & 62.8 & 2.9 & 20.0 \\
\hline
\end{tabular}

CP: compact cumulus; EX: expanded cumulus. h: hours of maturation before treatment with cycloheximide (total culture time 24 h); Mat: maturation control ( $24 \mathrm{~h}$ culture); Cyc-Mat: treatment with cycloheximide for $24 \mathrm{~h}$ followed by maturation for $24 \mathrm{~h}$; Direct: fixed directly upon removal from the follicle.

vesicle stage oocytes in the fluorescent nucleus configuration was significantly higher for CP than for EX oocytes ( $38 \%$ versus $9 \% ; P<0.05)$. There was no difference between EX and $\mathrm{CP}$ oocytes in the proportion of degenerating oocytes $(29 \%$ versus $31 \% ; P>0.1$.

\section{Ability of cycloheximide to inhibit nuclear maturation reversibly}

Cycloheximide was effective in suppressing maturation in both $\mathrm{EX}$ and $\mathrm{CP}$ oocytes, as there was no significant increase in maturing oocytes (diakinesis, MI and MII combined) between direct controls and $\mathrm{O} h$ suppression for either cumulus type $(P>0.1)$. There was no significant change in the proportion of germinal vesicle stage oocytes (combined fluorescent nucleus and condensed chromatin configurations) between direct controls and $\mathrm{O} h$ suppression for EX oocytes $(P>0.1)$. In the CP group, the proportion of oocytes in the fluorescent nucleus chromatin configuration decreased significantly between direct controls and $\mathrm{Oh}$ suppression treatment $(25 \%$ versus $0 \%$; $P>0.01$ ), whereas the proportion of oocytes in the condensed chromatin configuration did not ( $42 \%$ versus $33 \%$; $P>0.1$ ). Concomitant with the loss of fluorescent nucleus oocytes in the $\mathrm{CP}$ group after suppression, there was a significant increase in the proportion of degenerating oocytes $(P<0.05)$. The proportion of degenerating oocytes was significantly higher in the $\mathrm{CP}$ than in the EX group in the $0 \mathrm{~h}$ treatment $(P<0.01)$. In the EX group, there were no oocytes in the fluorescent nucleus configuration after $\mathrm{O} h$ suppression but this was not significantly different from the direct control $(6 \%$ versus $0 \% ; P>0.1$ ). The proportion of oocytes in the condensed chromatin stage was significantly higher in EX than in $\mathrm{CP}$ oocytes in the $\mathrm{Oh}$ suppression group $(P<0.05)$.
The suppression of maturation by cycloheximide in both EX and CP oocytes was completely reversible, as there was no difference between the proportion of oocytes reaching metaphase II in maturation controls and in suppressionmaturation treatments $(\mathrm{CP}, 21 \%$ versus $31 \%$; $\mathrm{EX}, 63 \%$ versus $63 \%$ respectively; $P>0.1$ ). In the maturation controls, a significantly greater proportion of $\mathrm{EX}$ than $\mathrm{CP}$ oocytes matured when only MII were compared $(P<0.01)$ or when MI and MII combined were compared $(P<0.01)$. In the suppressionmaturation treatment, a significantly greater proportion of EX than $\mathrm{CP}$ oocytes matured when comparing only MII $(P<0.01)$ or when comparing MI and MII combined $(P=0.01)$. In EX oocytes, there tended to be more abnormal metaphase configurations (chromosomes displaced from the metaphase plate or appearing to decondense) in the suppression-maturation treatment than in the maturation controls $(4 / 27$ versus $0 / 25$; $P=0.07)$. There were no abnormal configurations in metaphase $\mathrm{CP}$ oocytes in either the maturation or suppression-maturation treatments.

\section{Effect of addition of cycloheximide after $0,4,8,12$ or $16 \mathrm{~h}$ maturation}

For both CP and EX oocytes, there was no significant difference in the distribution of oocytes when cycloheximide was added after $4 \mathrm{~h}$ compared with at $\mathrm{Oh}(P>0.1)$. For the EX group, addition of cycloheximide at $8 \mathrm{~h}$ was associated with a significant decrease in the proportion of germinal vesicle stage oocytes $(P<0.01)$ and a significant increase in the proportion of oocytes in MI $(P<0.01)$ compared with at $0 \mathrm{~h}$. At $12 \mathrm{~h}$ and $16 \mathrm{~h}$, there was a significant increase in MII oocytes compared with at $\mathrm{Oh}(P<0.05$ and $P<0.01$, respectively) and the 
proportion of oocytes at MI tended to increase between $12 \mathrm{~h}$ and $16 \mathrm{~h}(P=0.052)$.

For $\mathrm{CP}$ oocytes, there was no significant change in the distribution until $12 \mathrm{~h}$, when the proportion of oocytes in the germinal vesicle stage decreased $(P<0.01)$ and the proportion in $\mathrm{MI}$ increased $(P<0.05)$ compared with the distribution at $0 \mathrm{~h}$. A significant increase in MII oocytes occurred at $16 \mathrm{~h}$ compared with at $0 \mathrm{~h}(P<0.05)$.

\section{Discussion}

The results of this study demonstrate that treatment with cycloheximide from the onset of culture inhibits the maturation of horse oocytes in vitro, and that this inhibition is reversible. An interesting feature of this study was the differential effect of cycloheximide treatment on different chromatin configurations in oocytes at the germinal vesicle stage. Horse oocytes possessing a germinal vesicle can have either diffuse fluorescence throughout the nucleus (the fluorescent nucleus configuration) or have chromatin condensed in only one area of the nucleus (condensed chromatin configuration; Hinrichs et al., 1993a). As seen in this study, the fluorescent nucleus configuration is more common in CP than in EX oocytes $(38 \%$ versus $9 \%$ of direct $\mathrm{GV}$ oocytes, respectively); this has been shown previously ( $31 \%$ versus $12 \%$ of direct GV oocytes, respectively; Hinrichs et al., 1993a). In the present study, the proportion of oocytes in the fluorescent nucleus configuration decreased from $25 \%$ in CP oocytes fixed directly upon removal from the follicle to $\% \%$ in $\mathrm{CP}$ oocytes after $24 \mathrm{~h}$ incubation with cycloheximide; however, the proportion of CP oocytes in the condensed chromatin configuration did not change. Despite the loss of the fluorescent nucleus oocytes after suppression, there was no difference in the proportion of oocytes reaching MIl between $\mathrm{CP}$ maturation controls and those maturing after suppression. This suggests that treatment with cycloheximide is associated with degeneration of oocytes in the fluorescent nucleus configuration, but not of oocytes in the condensed chromatin configuration. These data also suggest that oocytes in the fluorescent nucleus configuration do not contribute to the population of $\mathrm{CP}$ oocytes that reaches MII during maturation in vitro.

The superior performance of EX horse oocytes during maturation in vitro and related manipulations has been shown previously; EX oocytes matured to MII in equal (Zhang et al., 1989; Hinrichs et al., 1993a) or greater (Hinrichs et al., 1995a, b) proportions maintained viability better after suppression of maturation with follicular components (Hinrichs et al., 1995a) and were activated at higher rates after maturation in vitro (Hinrichs $e$ t al., 1995b) than were CP oocytes. This is in spite of the fact that EX oocytes are recovered from atretic follicles (Hinrichs, 1991; K. Hinrichs, unpublished).

EX horse oocytes mature more rapidly than do CP oocytes, reaching maximum proportions at MII by $24 \mathrm{~h}$ (Zhang et al., 1989; Hinrichs et al., 1993a), whereas the proportion of CP oocytes reaching metaphase II increases between $24 \mathrm{~h}$ and 30-32 h (Zhang et al., 1989; Hinrichs et al., 1993a; Alm and Torner, 1994). Correspondingly, in the present study, we found that EX oocytes required a shorter period for protein synthesis before becoming competent to undergo GVBD than did CP oocytes (maturation to MI: $8 \mathrm{~h}$ in EX versus $12 \mathrm{~h}$ in $\mathrm{CP}$; and maturation to MII: $12 \mathrm{~h}$ in EX versus $16 \mathrm{~h}$ in $\mathrm{CP}$ ).

The requirement for protein synthesis in relation to morphological stages of nuclear maturation for horse oocytes in this study appears to differ from that reported for bovine oocytes, in that addition of cycloheximide at $8 \mathrm{~h}$ to EX oocytes was associated with development of oocytes to $\mathrm{MI}$; however, GVBD has not occurred at this time (Hinrichs et al., 1993a). In cattle, development to MI does not result unless oocytes have matured in inhibitor-free media to, or past, the time GVBD would normally occur $(6.5 \mathrm{~h}$; Sirard et al., 1989; Kastrop et al., 1991; Tatemoto and Terada, 1995). Similarly, in goats, GVBD occurs between $2 \mathrm{~h}$ and $6 \mathrm{~h}$ of maturation, yet addition of cycloheximide at $6 \mathrm{~h}$ allows chromatin condensation only, without progression to MI (Pawshe et al. 1994). Our results for horses more closely parallel those for sheep, in which GVBD occurs at about $8 \mathrm{~h}$, and yet $50 \%$ of oocytes undergo GVBD and progression to MII when cycloheximide is added after $7 \mathrm{~h}$ of maturation (Moor and Crosby, 1986).

However, in horses as in other species, it appears that newly synthesized proteins are necessary for progression from MI to MII. These must include proteins necessary for the completion of meiosis I (extrusion of the polar body) as well as resumption of meiosis II. Formation of the MI plate is associated with high activity of MPF (histone $\mathrm{HI}$ kinase), as reported in pigs (Naito and Toyoda, 1991). Histone $\mathrm{HI}$ kinase activity decreases during the MI to MII transition in pigs. The proteins needed for progression from $\mathrm{MI}$ may therefore be related to deactivation of MPF (dephosphorylation of $\mathrm{P} 34 \mathrm{cdc} 2$ or proteolysis of cyclin B), or of cytostatic factor (CSF). CSF, which is a gamete-specific protein probably similar to the product of the gene, c-mos, stabilizes the cyclin-B component of MPF (O'Keefe et al., 1991). In mice, the c-mos product is required for resumption of meiosis II; however, oocytes injected with c-mos anti-sense oligonucleotides which block synthesis of the c-mos protein, do not remain in MI but extrude the polar body and form a pronucleus (O'Keefe et al., 1989). Arrest of oocytes at $\mathrm{MI}$ in the presence of cycloheximide suggests that MPF and CSF concentrations remain high when protein synthesis is inhibited.

In cattle and goat oocytes, addition of cycloheximide after maturation for $12 \mathrm{~h}$ or more is associated with abnormal metaphase configurations and pronucleus-like structures (Sirard et al., 1989; Pawshe et al., 1994). In cattle, $46 \%$ and $60 \%$ of oocytes form pronuclear structures when cycloheximide is added after $12 \mathrm{~h}$ or $16 \mathrm{~h}$ of maturation, respectively (Sirard et al., 1989). Similarly, in mice, addition of cycloheximide after MI leads to extrusion of a polar body and formation of a nucleus (Clarke and Masui, 1983). This led these authors to the conclusion that maintenance of MII requires protein synthesis. In our study, however, in the $12 \mathrm{~h}$ and $16 \mathrm{~h}$ cycloheximide treatments combined, only 1/69 CP oocytes and 3/65 EX oocytes formed pronuclear-type structures. This may relate to the refractoriness of matured horse oocytes to activation: ethanol, ethanol plus cycloheximide, or calcium ionophore alone did not significantly activate horse oocytes; however, treatment with calcium ionophore plus cycloheximide was effective in doing so (Hinrichs et al., 1995b). 
This work was supported by Deutsche Forschungsgemeinschaft (H. Alm) and the Massachusetts Horse Racing Research Fund (K. Hinrichs).

\section{References}

Alm $\mathbf{H}$ and Torner H (1994) In vitro maturation of horse oocytes Theriogenology 42 345-349

Clark HJ and Masui Y (1983) The induction of reversible and irreversible chromosome decondensation by protein inhibition during meiotic maturation of mouse oocytes Developmental Biology 97 291-301

Donahue RP (1968) Maturation of the mouse oocyte in vitro. I. Sequence and timing of nuclear progression Journal of Experimental Zoology 169 237-250

Fulka J Jr, Motlik J, Fulka J and Jilek F (1986) Effect of cycloheximide on nuclear maturation of pig and mouse oocytes Journal of Reproduction and Fertility 77 $281-285$

Hinrichs K (1991) The relationship of follicular atresia to follicle size, oocyte recovery rate on aspiration, and oocyte morphology in the mare Theriogenology 36 157-168

Hinrichs K, Schmidt AL, Friedman PP, Selgrath JP and Martin MG (1993a) In vitro maturation of horse oocytes: characterization of chromatin configuration using fluorescence microscopy Biology of Reproduction 48 363-370

Hinrichs K, Schmidt AL and Selgrath JP (1993b) Atlas of chromatin configurations of germinal vesicle-stage and maturing horse oocytes Equine Veterinary Journal Supplement 15 60-63

Hinrichs K, Martin MG, Schmidt AL and Friedman PP (1995a) Effect of follicular components on meiotic arrest and resumption in horse oocytes Journal of Reproduction and Fertility 104 149-156

Hinrichs K, Schmidt AL and Selgrath JP (1995b) Activation of horse oocytes Biology of Reproduction Monograph 1 319-324

Kastrop PMM, Bevers MM, Destree OHJ and Kruip ThAM (1990) Analysis of protein synthesis in morphologically classified bovine follicular oocytes before and after maturation in vitro Molecular Reproduction and Development $26222-226$

Kastrop PMM, Hulshof SCJ, Bevers MM, Destree OHJ and Kruip ThAM (1991) The effect of $\alpha$-amanitin and cycloheximide on nuclear progression, protein synthesis, and phosphorylation during bovine oocyte maturation in vitro Molecular Reproduction and Development 28 249-254

McGaughey RW and Van Blerkom J (1977) Pattern of polypeptide synthesis of porcine oocytes during maturation in vitro Developmental Biology 56 $241-254$

Moor RM and Crosby IM (1986) Protein requirements for germinal vesicle breakdown in ovine oocytes Journal of Embryology and Experimental Morphology 94 207-220
Motlik J and Fulka J, Jr (1976) Breakdown of the germinal vesicle in pig oocytes in vivo and in vitro Journal of Experimental Zoology 198 155-162

Naito K and Toyoda $Y$ (1991) Fluctuation of histone H 1 kinase activity during meiotic maturation in porcine oocytes Journal of Reproduction and Fertility $\mathbf{9 3}$ 467-473

O'Keefe SJ, Wolfes H, Kiessling AA and Cooper GM (1989) Microinjection of antisense c-mos oligonucleotides prevents meiosis II in the maturing mouse egg Proceedings of the National Academy of Sciences USA $867038-$ 7042

O'Keefe SJ, Kiessling AA and Cooper GM (1991) The c-mos gene product is required for cyclin $B$ accumulation during meiosis of mouse eggs Proceedings of the National Academy of Sciences USA 88 7869-7872

Pawshe CH, Appa Roa KBC, Jain SK and Totey SM (1994) Biochemical studies on goat oocytes: timing of nuclear progression, effect of protein inhibitor and pattern of polypeptide synthesis during in vitro maturation Theriogenology 42 307-320

Pincus G and Enzmann EV (1935) The comparative behaviour of mammalian eggs in vivo and in vitro. I. The activation of ovarian eggs Journal of Experimental Medicine 62 665-675

Schultz RM and Wassarman PM (1977) Biochemical studies of mammalian oogenesis: protein synthesis during oocyte growth and meiotic maturation in the mouse Journal of Cell Science 24 167-194

Sirard MA (1990) Temporary inhibition of in vitro meiotic resumption by adenylate cyclase stimulation in immature bovine oocytes Theriogenology 33 757-767

Sirard MA and First NL (1988) In vitro maturation of oocyte nuclear maturation in the bovine Biology of Reproduction 39 229-234

Sirard MA, Florman HM, Leibfried-Rudledge ML, Barnes FL, Sims ML and First NL (1989) Timing of nuclear progression and protein synthesis necessary for meiotic maturation of bovine oocytes Biology of Reproduction 40 12571263

Stern S, Rayyis A and Kennedy JF (1972) Incorporation of amino acids during maturation in vitro by mouse oocytes: effect of puromycin on protein synthesis Biology of Reproduction 7 341-346

Tatemoto H and Terada T (1995) Time-dependent effects of cycloheximide and $\alpha$-amanitin on meiotic resumption and progression in bovine follicular oocytes Theriogenology 43 1107-1113

Wassarman PM, Josefowicz WJ and Letourneau GE (1976) Meiotic maturation of mouse oocytes in vitro: inhibition of maturation at specific stages of nuclear progression Journal of Cell Science 22 531-545

Zhang JJ, Boyle MS, Allen WR and Galli C (1989) Recent studies on in vivo fertilization of in vifro matured horse oocytes Equine Veterinary Journal, Supplement 8 101-104 